\title{
Regulation of Bmp4 Expression in Odontogenic Mesenchyme: From Simple to Complex
}

\author{
Hui Kong ${ }^{\mathrm{a}}$ Ying Wang ${ }^{\mathrm{b}}$ Manshi Patel $^{\mathrm{a}} \quad$ Gabriele Mues $^{\mathrm{a}}$ Rena N. D'Souza ${ }^{\mathrm{a}}$ \\ ${ }^{a}$ Department of Biomedical Sciences, Texas A\&M University Health Science Center Baylor College of Dentistry, \\ Dallas, Tex., and ${ }^{b}$ Department of Orthodontics, School of Dental Medicine, University at Buffalo, State University of \\ New York, Buffalo, N.Y., USA
}

\section{Key Words}

Bmp4 expression • Pax9 • Msx1 - Tooth bud mesenchyme • Tooth agenesis

\begin{abstract}
For many years the molecular mechanisms governing bone morphogenetic protein 4 (Bmp4) expression in tooth bud mesenchyme could be explained by an uncomplicated model involving the interaction of the homeobox gene Msx1 and the paired domain gene Pax 9 and a limited proximal promoter segment of Bmp4. New insights have led to major revisions, but we are still far from understanding the role of Msx 1 and Pax9 in the complex processes that result in the expression of Bmp4 in the mesenchymal layer of the developing tooth bud. The objective of these studies was to gain further insight into the molecular relationship between Pax9, Msx1, and Bmp4 in dental mesenchyme and explore its association with nonsyndromic tooth agenesis in humans.
\end{abstract}

Copyright $\odot 2011$ S. Karger AG, Basel

\section{Introduction}

Mesenchymal expression of the signaling factor bone morphogenic protein 4 (Bmp4) is essential for the progression of tooth development. More than a decade ago, studies with knockout mice revealed that Bmp4 expression in tooth bud mesenchyme requires the homeodomain protein Msx 1 and the paired domain transcription factor Pax9; homozygous deletion of either protein or heterozygous deficiency of both resulted in the arrest of tooth development which could be partially rescued by Bmp4 overexpression [Chen et al., 1996; Peters et al., 1998; Bei et al., 2000; Nakatomi et al., 2010]. In vitro investigations of the molecular basis for the Pax9/Msx1/ Bmp4 interrelationship offered a straightforward explanation for the observed facts in that Pax 9 activated the Bmp4 promoter directly, while the Msxl protein potentiated the activity of Pax9 but had only inhibitory activity by itself [Ogawa et al., 2006]. New findings about the vast size and complexity of the Bmp4 regulatory area [Chandler et al., 2009] as well as a more detailed analysis of the

\section{Abbreviations used in this paper}

Bmp4 bone morphogenetic protein 4

Dlx1, 2, 5 distal-less homeobox gene 1, 2, or 5

Gro/Tle Groucho/transducin-like enhancer of split

$\mathrm{kb} \quad$ kilo-base pairs

Msx1, 2 homolog of muscle segment homeobox gene,

drosophila, 1 or 2

Pax9 paired box gene 9

\section{KARGER}

Fax +4161306 1234

E-Mail karger@karger.ch

www.karger.com
(C) 2011 S. Karger AG, Basel

Accessible online at: www.karger.com/cto
Dr. Rena N. D'Souza

Department of Biomedical Sciences

Texas A\&M University Health Science Center Baylor College of Dentistry

3302 Gaston Ave., Dallas, TX 75246 (USA)

Tel. +1 214828 8260, E-Mail rdsouza@bcd.tamhsc.edu 
Pax9 and Msx1 molecular interactions using wild-type and mutant proteins have led to a revision of the original theory about Bmp4 regulation in tooth bud mesenchyme. In this paper, we present results of our recent studies that further explored the molecular relationship between Pax9, Msx1, and Bmp4 expression as well as the relationship of Pax9 with other mesenchymally expressed transcription factors. Our results suggest that the molecular mechanisms that underlie Bmp4 activation in tooth bud mesenchyme are indeed complex and cannot fully explain the role of Msx1 in normal and abnormal tooth development.

\section{Materials and Methods}

\section{Coimmunoprecipitation}

Msx1, Msx2, Dlx1, Dlx2, and Dlx 5 coding areas were cloned into Flag-tagged pCMV expression vectors (Stratagene); the Pax9 coding region was cloned into a Myc-tagged vector. For coimmunoprecipitation, Pax9- and homeodomain protein-containing vectors were cotransfected (Fugene; Roche) into COS7 cells. Cell lysates were immunoprecipitated with anti-Flag antibodies followed by Western blot with anti-Pax9 antibody.

\section{Promoter Reporter Assays}

Bmp4 promoter fragments were PCR amplified and subcloned into pGL3 luciferase reporter vectors (Promega) containing a $0.3-\mathrm{kb}$ proximal Bmp4 promoter segment which served as the minimal baseline promoter. The promoter constructs were cotransfected with increasing amounts of Msx1 expression plasmids $(0.0,0.25,0.5$, and $1 \mu$ g supplemented with $1.0,0.75,0.5$, and $0.0 \mu \mathrm{g}$ of empty expression vector, respectively) into COS7 cells and tested for luciferase activity. A pCMV-SPORT b-gal plasmid was used as the internal control. Chandler et al. [2009] kindly provided a BAC clone spanning the upstream 200-kb Bmp4 promoter section.

\section{Cell Culture}

Cos7 cells were cultured in DMEM (Invitrogen, USA) supplemented with $10 \%$ fetal calf serum, penicillin (100 units $/ \mathrm{ml})$, and streptomycin $(100 \mathrm{mg} / \mathrm{ml})$. Cells were transfected with plasmids using FuGENE HD (Roche) according to the manufacturer's instructions.

\section{ChIP-PCR}

Proteins in dissected molar tooth buds of E14.5 CD1 mouse embryos were cross-linked to chromatin with $1 \%$ formaldehyde/1 $\times$ PBS. After enzymatic shearing of the chromatin, a mixture of Pax9 7C2 (Abcam) and M18 (sc-7746; Santa Cruz) antibodies was used to collect antibody-bound protein/DNA complexes using protein $G$ agarose beads. Pax9-bound DNA was purified and analyzed by PCR with primers specific for the Bmp4 promoter. Chromatin precipitated by $\operatorname{IgG}$ was used as the negative control.

\section{Results}

\section{Pax9-Msx1 Physical and Functional Protein}

\section{Interaction}

We have previously shown that Pax9 and Msx1 can be coimmunoprecipitated, suggesting that both proteins interact physically in order to achieve their synergistic effect on Bmp4 activation. In order to test if this physical interaction is necessary we took advantage of the properties of the related Msx2 protein which is also expressed in tooth bud mesenchyme and shows a similar synergistic effect with Pax9 on Bmp4 expression. The homeodomain of Msx2 differs by only 2 amino acids from the Msx1 homeodomain (98\% identity), but the rest of the 2 Msx proteins is quite dissimilar, with only $45 \%$ identity. Testing Msx2 for coimmunoprecipitation with Pax9 yielded a negative result (fig. 1a), implying that (a) the protein-protein interaction as demonstrated by coimmunoprecipitation is not involved in the functional synergism with Pax9 and (b) the homeodomain is not responsible for the coimmunoprecipitation of Pax9 and Msx1 but is involved in the functional synergism with Pax9. One Msx1 homeodomain mutant with abolished DNA binding was tested for synergism with Pax9 and found to be even more active than wild-type Msx1 [Wang et al., 2011], proving that DNA binding by the Msx1 homeodomain is not necessary for the synergistic effect. These experiments also revealed that Msx2 is a more efficient potentiator of Pax9-induced Bmp4 activation than Msx1 when human instead of mouse Bmp4 promoter sequences are used (fig. 1b).

\section{Pax9-Bmp4 Promoter Interaction}

Pax9 is unquestionably effective in activating the proximal Bmp4 promoter in vitro, but does this translate to relevance in vivo? A first step was to test if Pax9 is bound to the Bmp4 promoter in mouse embryonic tooth bud mesenchyme. We performed a ChIP-PCR assay using chromatin from E14 tooth buds and a mix of 2 Pax 9 antibodies to precipitate Pax9-bound DNA fragments. IgGprecipitated fragments served as the negative control. Figure 2 shows that PCR with Bmp4 promoter-specific primers could amplify several Bmp4 promoter fragments from Pax9-precipitated chromatin but not from IgG-precipitated chromatin, suggesting that Pax9 is indeed bound to the proximal Bmp4 promoter during tooth development.

\section{Msx1-Bmp4 Promoter Interaction}

Our previous experiments demonstrated that the proximal 3-kb Bmp4 promoter sequence was unresponsive to Msx1; Msx1 could only potentiate Pax9-induced 
Fig. 1. a Expression vectors containing Pax9 and Flag-tagged Msx1, Msx2, Dlx1, Dlx2, or Dlx5 were cotransfected into Cos7 cells. Whole cell lysates were treated with anti-Flag-agarose for immunoprecipitation (IP). Western blots of the IP products are shown. Upper panel: Western blot of IP with anti-Flag; lower panel: Western blot of IP with anti-pax9 antibody. Only Msx1 is coimmunoprecipitated with Pax9. b Msx2 shows greater potentiation of the Pax9-induced Bmp4 promoter activation than Msx1 when using a human Bmp4 promoter fragment (hBmp4). Statistical evaluation of expression differences: Pax9 versus vector, $\mathrm{p}=0.002 ; \mathrm{Msx} 1+\mathrm{Pax} 9$ versus Msx1+vector, $\mathrm{p}=0.000001 ; \mathrm{Msx} 2+\mathrm{Pax} 9$ versus Msx2+vector, $\mathrm{p}=0.000064 ; \mathrm{Msx} 2+$ Pax9 versus Msx1+Pax9, $\mathrm{p}=0.002023$. c Most of the fragments from the upstream extended Bmp4 regulatory region have a negative effect on expression (not all tested fragments are shown); only the -42 - to -47-kb region and the 668-kb ECR from this region show increased activity. The statistical significances of the expression differences between the vector and the 3 different Msx1 concentrations are as follows: $-97-$ to $-87-\mathrm{kb}$ fragment: 0.044, 0.049 , and 0.013 ; -87 - to $-77-\mathrm{kb}$ fragment: $0.029,0.018$, and $0.003 ;-77-$ to $-67-\mathrm{kb}$ fragment: $0.204,0.206$, and 0.106 ; $-67-$ to -57-kb fragment: $0.963,0.021$, and 0.002 ; -47- to -42-kb fragment: $0.586,0.025$, and 0.002 , and ECR2-668 bp: $0.679,0.560$, and 0.001 .

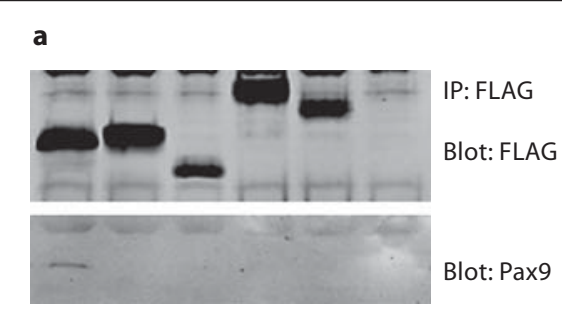

Msx1 Msx2 Dlx1 Dlx2 Dlx5 Vector

c

pGL3-mp-mBmp4-10 kb \#1 (-97 kb to $-87 \mathrm{~kb}$ ) cotransfected with increasing amounts of Msx1

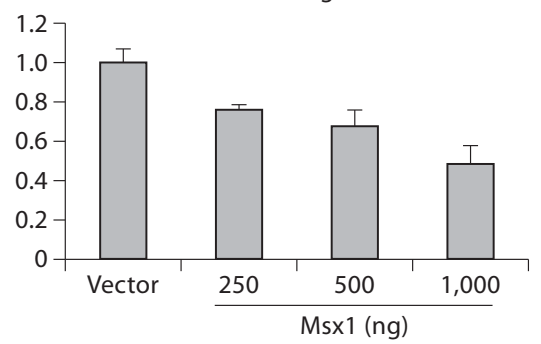

pGL3-mp-mBmp4-10 kb \#3 (-77 kb to $-67 \mathrm{~kb}$ ) cotransfected with increasing amounts of Msx1

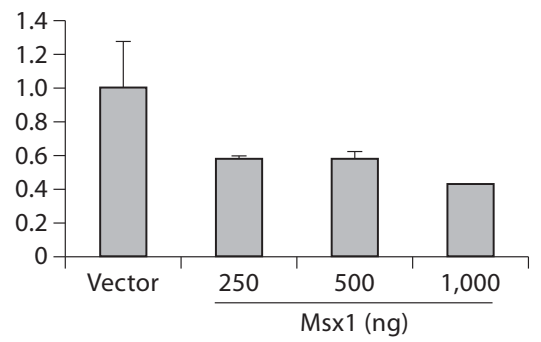

pGL3-mp-mBmp4-5 kb \#3 (-47 kb to $-42 \mathrm{~kb}$ ) cotransfected with increasing amounts of Msx1

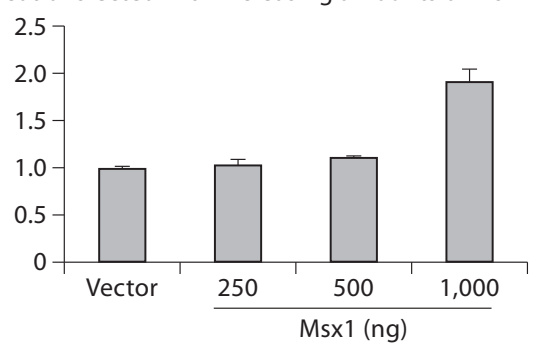

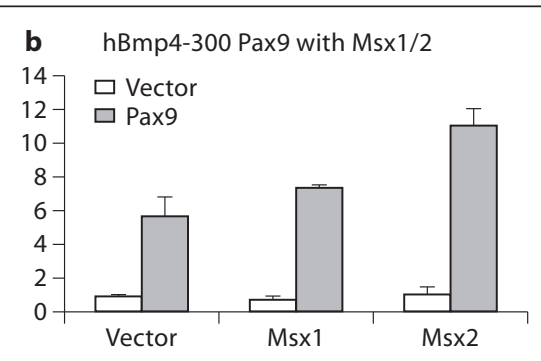

pGL3-mp-mBmp4-10 kb \#2 (-87 kb to -77 kb) cotransfected with increasing amounts of Msx1

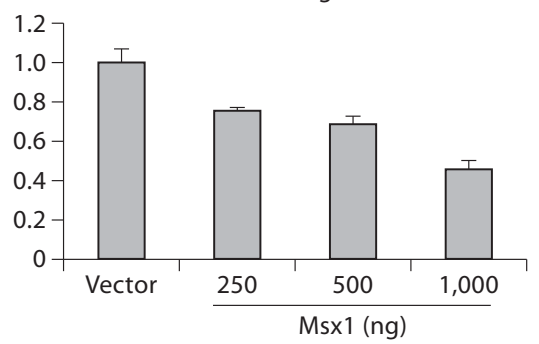

pGL3-mp-mBmp4-10 kb \#4 (-67 kb to $-57 \mathrm{~kb}$ ) cotransfected with increasing amounts of Msx 1

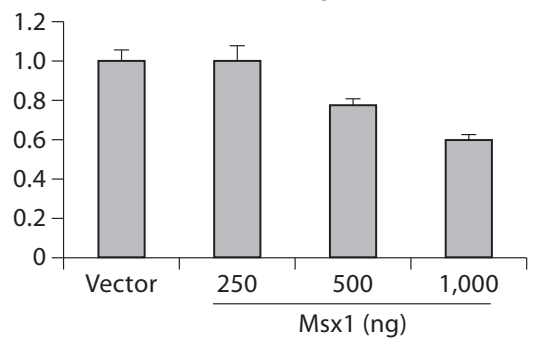

pGL3-mp-mBmp4-ECR2-668 bp cotransfected with increasing amounts of Msx1

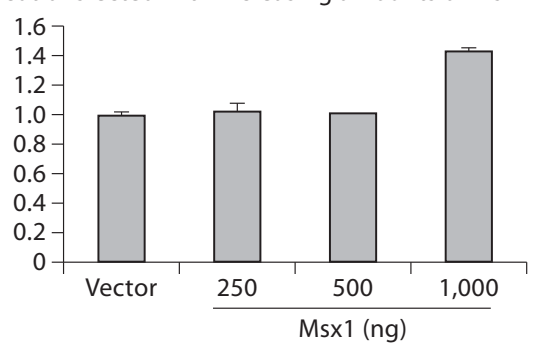

activation of the Bmp4 promoter. However, recently, Chandler et al. [2009] reported that the regulatory area of Bmp4 extends from about $200 \mathrm{~kb}$ upstream to $200 \mathrm{~kb}$ downstream of the Bmp4 coding area. They also found that enhancer elements for the tooth bud mesenchyme expression of Bmp4 reside in an upstream extended segment somewhere between -28 and $-199 \mathrm{~kb}$. This discovery made it quite obvious that a much larger segment of the promoter region of Bmp4 had to be tested for respon- siveness to Msx1 to exclude a direct activation. We subcloned the proximal $97 \mathrm{~kb}$ of the extended Bmp4 upstream promoter sequence in 5 - to $10-\mathrm{kb}$ segments into pGL3 reporter vectors and cotransfected each subclone with increasing amounts of an Msxl expression vector into COS7 cells. Figure 1c shows that Msx1 acted as an inhibitor on all of the segments except the -47 - to $-42-\mathrm{kb}$ fragment, the same area that had been described by Chandler et al. [2009] as an evolutionary conserved en- 


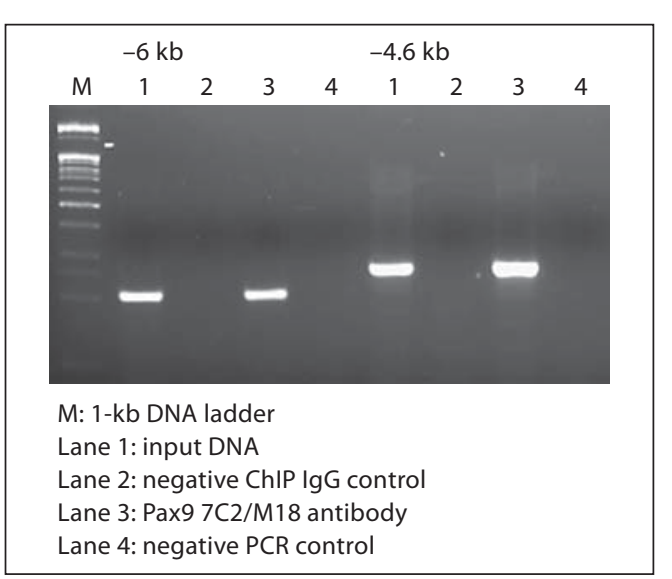

Fig. 2. ChIP-PCR results with chromatin precipitated from E14 mouse tooth buds by a mix of Pax9 antibodies or by IgG. Chromatin precipitated with Pax9 antibodies can be amplified with primers to Bmp4 promoter sequences located at -4.6 and $-6 \mathrm{~kb}$ (lanes 3), while chromatin precipitated by IgG are not amplified by these primers (lanes 2 ). Lanes 1 and lanes 4 are the positive and negative controls, respectively.

hancer element (ECR2)-containing sequence. The isolated 0.67-kb ECR2 enhancer sequence showed a similar effect, while Pax9 was inactive or even inhibitory.

\section{Discussion}

Initial investigations of the molecular mechanisms leading to Bmp4 activation by Pax9 and Msx1 strongly suggested a model in which Pax9 directly interacts with the Bmp4 promoter, thus enhancing its transcription. Pax9 also activates the expression of Msx1 protein which subsequently cooperates with Pax9 to amplify Bmp4 transcription. This model also explained the fact that Msx1 by itself was unable to activate Bmp4 expression.

However, further investigations of the molecular interactions between the Bmp4 regulatory region, $\mathrm{Pax} 9$, and Msx 1 as well as advancing knowledge about the complexities of gene regulation raised questions about the original model. Investigations by Chandler et al. [2009] localized the regulatory region of Bmp4 for tooth bud mesenchyme expression to enhancer elements more than $28 \mathrm{~kb}$ upstream of the Bmp4 coding region, which is far beyond the promoter region that had been tested.

Some of our molecular interaction studies strengthened our previous model. The Pax9 protein does indeed bind to the proximal Bmp4 promoter in mouse E14 tooth buds according to our ChIP-PCR results. In our investi- gations about the mechanisms of action of human tooth agenesis-causing Pax9 mutations [Wang et al., 2009] we showed that loss of DNA binding, accompanied by corresponding reductions of reporter gene expression, appears to be the pathogenic effect of these Pax 9 mutants. There remains some uncertainty about our results because the DNA region which was tested in these assays spans the proximal $3 \mathrm{~kb}$ of the Bmp 4 regulatory area and not the upstream segments described by Chandler et al. [2009]. However, it may be possible that in tooth bud mesenchyme Pax9 is a constitutive occupant of the proximal core promoter region which is then targeted by tissuespecific, active enhancer elements from 28 to $200 \mathrm{~kb}$ upstream to form a viable transcription factor complex.

The interaction between Pax9 and Msx1 has become less clear. Coimmunoprecipitation of Pax9 and Msx1 is not likely to be mediated by the homeodomain of Msx1, and the molecular interaction causing coimmunoprecipitation is not the physical basis for the synergism between Pax9 and Msx1; to the contrary, it may reflect the ability of Msx1 to regulate Pax9 protein levels by sequestering Pax9. In vitro tests suggest that the homeodomain of Msx1 (and Msx2) appears to be necessary for the synergistic effect with Pax9, but homeodomain-mediated DNA binding is not required for synergism with Pax9. We recently showed that tooth agenesis-causing Msx1 mutations do not abolish synergism with Pax9 [Wang et al., 2011], and we reached the conclusion that Msx1 must use an alternative or additional mechanism to activate Bmp4. One tooth agenesis-causing Msxl mutation which is located in a Gro/Tle-binding site of Msxl points to a disturbance in transcriptional repression mechanisms [RaveHarel et al., 2005] instead of transcriptional activation.

Direct activation of Bmp4 by Msxl is unlikely, but it has not been excluded yet because the ECR enhancer in the extended Bmp4 regulatory area responded positively in contrast to the remainder of the $98 \mathrm{~kb}$ of tested Bmp4 regulatory area. Overall, the previously established plain and coherent model is no longer supported by these new observations that suggest a high degree of complexity in the physiologic roles and molecular relationship between genes involved in Bmp-mediated signaling during tooth morphogenesis.

\section{Acknowledgement}

This work was supported by National Institutes of Health/National Institute of Dental and Craniofacial Research (NIH/NIDCR) R01 award DE019471-01 to R.N.D’S. 


\section{References}

Bei, M., K. Kratochwil, R.L. Maas (2000) BMP4 rescues a non-cell-autonomous function of Msx1 in tooth development. Development 127: 4711-4718.

-Chandler, K.J., R.L. Chandler, D.P. Mortlock (2009) Identification of an ancient Bmp4 mesoderm enhancer located $46 \mathrm{~kb}$ from the promoter. Dev Biol 327: 590-602.

-Chen, Y., M. Bei, I. Woo, I. Satokata, R. Maas (1996) Msx1 controls inductive signaling in mammalian tooth morphogenesis. Development 122: 3035-3044.
Nakatomi, M., X.P. Wang, D. Key, J.J. Lund, A. Turbe-Doan, R. Kist, A. Aw, Y. Chen, R.L. Maas, H. Peters (2010) Genetic interactions between Pax9 and Msx1 regulate lip development and several stages of tooth morphogenesis. Dev Biol 340: 438-449.

Ogawa, T., H. Kapadia, J.Q. Feng, R. Raghow, H. Peters, R.N. D'Souza (2006) Functional consequences of interactions between $\operatorname{Pax} 9$ and Msx1 genes in normal and abnormal tooth development. J Biol Chem 281: 18363-18369.

Peters, H., A. Neubuser, K. Kratochwil, R. Balling (1998) Pax9-deficient mice lack pharyngeal pouch derivatives and teeth and exhibit craniofacial and limb abnormalities. Genes Dev 12: 2735-2747.
Rave-Harel, N., N.L. Miller, M.L. Givens, P.L. Mellon (2005) The Groucho-related gene family regulates the gonadotropin-releasing hormone gene through interaction with the homeodomain proteins MSX1 and OCT1. J Biol Chem 280: 30975-30983.

Wang, Y., J.C. Groppe, J. Wu, T. Ogawa, G. Mues, R.N. D’Souza, H. Kapadia (2009) Pathogenic mechanisms of tooth agenesis linked to paired domain mutations in human PAX9. Hum Mol Genet 18: 2863-2874.

Wang, Y., H. Kong, G. Mues, R. D’Souza (2011) Msx1 mutations: How do they cause tooth agenesis? J Dent Res 90: 311-316. 